\title{
ETHICAL DILEMMA OF CESAREAN SECTION ON MATERNAL REQUEST (CSMR)
}

\author{
Abeera Choudry, Asifa Siraj, Humaira Tariq*, Faiqa Chugtai, Uzma Urooj \\ Pak Emirates Military Hospital/National University of Medical Sciences (NUMS) Rawalpindi Pakistan, *Combined Military Hospital/National University of \\ Medical Sciences (NUMS) Rawalpindi Pakistan
}

\begin{abstract}
Objective: To study the demographic characteristics of pregnant ladies and factors contributing towards rise in cesarean section on maternal request to aid the obstetricians in decision making.

Study Design: Cross sectional analytical survey.

Place and Duration: Gynecology Department of Pak Emirates Military Hospital, Rawalpindi, from Nov 2019 to Mar 2020.

Methodology: One hundred and fifteen women of child bearing age requesting cesarean section were included in the study. Demographic details were noted. A study proforma was filled for determinants of primary and secondary tocophobia and factors that may be improved for vaginal delivery.

Results: A total of 115 patients with mean age of 27.99 years were included. Amongst them, 88 (76.5\%) were Punjabi with 92 $(80 \%)$ living in rural area. Primigravida were $11(9.6 \%), 83(72.2 \%)$ had previous lower segment cesarean section and $3(2.6 \%)$ had vaginal delivery. For primary tocophobia, 22 ( $24.4 \%)$ experienced anxiety. Fear of labor pains was seen in 20 (19.2\%) and lack of control in 27 (26\%). For secondary tocophobia, 15 (37.5\%) were fearful of prolonged labor and 5 (22.5\%) of sub optimal birth outcome. In women with previous one cesarean section, $13(14.8 \%)$ correlated negatively with birth experience and 20 $(22.7 \%)$ found timed cesarean section convenient. For vaginal delivery, pain relief was preferred by 19 (20.2\%) and 31 (33\%) wanted pain relief and attendant.

Conclusion: Better understanding of fears behind maternal request for cesarean section can lead to improved attitudes towards vaginal delivery. The negative perceptions of pregnant ladies should be addressed in antenatal visits.
\end{abstract}

Keywords: Cesarean section, Maternal request, Tocophobia.

\footnotetext{
This is an Open Access article distributed under the terms of the Creative Commons Attribution License (http://creativecommons.org/licenses/by/4.0), which permits unrestricted use, distribution, and reproduction in any medium, provided the original work is properly cited.
}

\section{INTRODUCTION}

Rising cesarean section rate is a globally debatable issue. There are serious concerns regarding woman's obstetric future after cesarean section delivery and neonatal respiratory distress and prematurity. As per World Health Organization (WHO) recommendations, medical interventions must be kept to minimum in maternal and child health care. The cesarean section rate should not rise above $10-15 \% 1$. According to $\mathrm{WHO}$, Cesarean section rates have risen from $2.7 \%$ in $1990-91$ to $15.8 \%$ in $2012-13$ with around 9700 mortalities due to maternal complications in 2015 in Pakis$\tan ^{2,3}$. A significant rise of up to $40 \%$ has been seen in rich, educated and urban living ladies wishing for a reduced family size, avoidance of pain and unpredictability of normal labor and damage to pelvic floor. In Pakistan, medically non- indicated cesarean sections have risen in the last decade. The education provides higher autonomy to the females to take their own decisions regarding child birth. Cesarean section is a key predicator of accessibility of health care services to women. About $11.5 \%$ of rural women had cesarean section as compared to $26.5 \%$ of urban ladies. WHO

Correspondence: Dr Humaira Tariq, Consultant Gynecologist, Combined Military Hospital, Rawalpindi Pakistan

Received: 06 Oct 2020; revised received: 10 Feb 2020; accepted: 11 Feb 2021 released a new statement in 2015 stating that the rate of cesarean section should not exceed $10 \%$ and should not be less than $5 \%$ as both extremes can have adverse impact on maternal health and quality of life ${ }^{4}$.

Cesarean section on maternal request (CSMR) is defined as a planned cesarean section performed on maternal request in the absence of indications for cesarean section and contraindications to vaginal delivery ${ }^{5}$. The causes for increased cesarean sections are multi factorial. Traditionally it was considered inappropriate to perform cesarean section without a clinical indication. However, a rising trend has been observed in cesarean sections performed on demand or for nonevidence based reason. CSMR is not a well recognized and investigated entity. It is affected by a complex labyrinth of health care providers, health system, patients, culture, beliefs, fashion and social media. Most obstetricians have faced this request from pregnant women in their clinical practice. There is a lack of explicit data and surveys regarding the incidence and impact of rising rate of CSMR. In USA, the exact incidence is not known but it is estimated that CSMR occurs in less than $3 \%$ of all the deliveries. ACOG Committee Opinion on CSMR recommends that women requesting CSMR should undergo through assessment in terms of risk factors, future pregnancy plans, social and cultural 
context $^{6}$. If no other indications are present, then CSMR should not be performed before 39 weeks of gestation ${ }^{7}$. The rise in cesarean section rate in Pakistan is similar to other developing countries and is primarily due to ceserean sections performed for non-evidence based reasons like professional convenience and maternal request ${ }^{8}$.

Tocophobia is defined as an intense fear of childbirth and is the leading psychological cause of CSMR. Primary tocophobia is morbid fear of childbirth in a woman, who has had no previous experience of pregnancy. Secondary tocophobia is experienced by women who had a previous traumatic birth experience leading to phobia of child birth. Women with tocophobia either avoid pregnancy or request cesarean section for child birth $^{9}$. Proponents debate that elective cesarean section cannot guaranteenormality but it can avoid the expected morbidities related to vaginal birth. This has changed the trends in affluent strata of the societies. In London, $31 \%$ of the female obstetricians with uncomplicated pregnancies chose cesarean section over normal delivery for themselves ${ }^{10}$. These women are not the representative of the common population. Nowadays obstetricians are at a turning point because of the advancements that have made cesareans safe and the substantial morbidity of vaginal delivery that cannot be negated.

There is a need to look into the rationale and experiences behind the maternal request for cesarean section to understand and overcome the factors leading to rise in CSMR. There is a strong ethical dilemma behind rising CSMR. At one end of the spectrum is the unpredictability of normal labor surrounding the maternal fears. While at the other end is the rise in cesarean sections performed for non-medical reasons. The obstetrician has to balance the two ends and formulate a safe plan for delivery and health of the mother. Respectful maternity care demands provision of physical and mental support during labor to ease the process of natural childbirth.

This study was conducted to gather information to find out the perceptions of women of childbearing age regarding tocophobias leading to rise in CSMR. The purpose of this study was to estimate the burden of the problem so that proper guidelines can be set for identification and timely intervention to reduce the rate of CSMR and encourage natural childbirth.

\section{METHODOLOGY}

A cross sectional survey was carried out for duration of three months from November 2019 to
February 2020. It was conducted at Obstetrics and Gynecology Department, Pak Emirates Military Hospital, Rawalpindi. The study was formally approved by the ethical research review committee (IERB certificate number A/28/EC/220/2020) and informed consent was taken from all theparticipants.

Non-probability consecutive sampling technique was used. A total of 115 woman of child bearing age willing to participate were included in the study. Sample size was calculated by using Open Epi calculator. The prevalence of tocophobia was found to be $7.5 \%$ in one of the studies 7 . Those women who had co morbidities affecting mode of delivery, had completed their families and were not of childbearing age were excluded from thestudy.

A study proforma was designed for the determinants of primary and secondary tocophobia. It also included suggestions for improving experience of normal labor like provision of pain relief and availability of a companion during labor. The demographic data of the women was noted including age, socio economic status, education, entitlement, ethnicity and past obstetric history.

The data was analyzed using SPSS-23. The counts with the percentages were given for baseline characteristics including entitlement or non-entitlement for army hospital, socioeconomic class, education and other studied factors. Descriptive analysis was done to find out the common factors of primary and secondary tocophobia among the samples.

\section{RESULTS}

The data of 115 women was analyzed. Out of these, $107(93 \%)$ were entitled and $8(7 \%)$ were private patients. Mean age in years was $27.99 \pm 3.24$. Lower middle class was the most common socio economic group $85(73.9 \%)$ while 15 (13\%) each belonged to upper and lower socio economic group. Ethnicity was predominantly Punjabi 88 (76.5\%) with $92(80 \%)$ living in rural and $23(20 \%)$ in urban areas. The educational status ranged from middle $34(29.6 \%)$ to matric 33 (28.7\%). The bachelor's degree was held by 14 (12.2\%). Amongst these ladies, 83 (72.2\%) had cesarean section, $3(2.6 \%)$ had vaginal delivery and $17(14.8 \%)$ experienced both vaginal delivery and cesarean section. Tocophobia was expressed by 80 (69.6\%) women. These demographic characteristics are shown in table-I.

Primary tocophobia was divided into social factors and fear of child birth. In the social factors, 25 $(27.8 \%)$ felt that they were too weak for labor, 22 
(24.4\%) felt anxiety and $16(17.8 \%)$ feared abuse and trauma. Family and friends recommended cesarean section for $22(24.4 \%)$ participants. Amongst the factors influencing the fear of child birth, 27 (26\%) ladies expressed lack of control, 20 (19.2\%) feared intense prolonged pain, 15 (14.4\%) each feared emergency cesarean section and loss of baby. This is shown in table-II.

Table-I: Demographic characteristics $(n=115)$.

\begin{tabular}{|c|c|c|}
\hline \multicolumn{2}{|l|}{ Characteristics } & n (\%) \\
\hline \multirow{2}{*}{ Entitlement } & Entitled & $107(93)$ \\
\hline & Private & $8(7)$ \\
\hline \multirow{3}{*}{$\begin{array}{l}\text { Socioeconomic } \\
\text { Class }\end{array}$} & Lower & $15(13)$ \\
\hline & Lower middle & $85(73.9)$ \\
\hline & Upper middle & $15(13)$ \\
\hline \multirow{2}{*}{ Residence } & Rural Area & $92(80)$ \\
\hline & Urban Area & $23(20)$ \\
\hline \multirow{4}{*}{ Ethnicity } & Punjabi & $88(76.5)$ \\
\hline & Pathan & $14(12.2)$ \\
\hline & Kashmiri & $10(8.7)$ \\
\hline & Saraiki & $3(2.6)$ \\
\hline \multirow{5}{*}{$\begin{array}{l}\text { Educational } \\
\text { status }\end{array}$} & Primary & $19(16.5)$ \\
\hline & Middle & $34(29.6)$ \\
\hline & Matric & $33(28.7)$ \\
\hline & F.Sc. & $15(13)$ \\
\hline & Bachelors & $14(12.2)$ \\
\hline \multirow{5}{*}{$\begin{array}{l}\text { Obstetric } \\
\text { History }\end{array}$} & Previous SVDs & $3(2.6)$ \\
\hline & LSCS & $83(72.2)$ \\
\hline & Assisted Vaginal Delivery & $1(0.9)$ \\
\hline & SVD +LSCS & $17(14.8)$ \\
\hline & PG & $11(9.6)$ \\
\hline \multirow{2}{*}{ Tocophobia } & Yes & $80(69.6)$ \\
\hline & No & $35(30.4)$ \\
\hline Age (years) & Mean \pm SD & $27.99 \pm 3.24$ \\
\hline \multicolumn{3}{|c|}{ Table-II: Primary tocophobic factors. } \\
\hline \multicolumn{2}{|c|}{ Factors } & n (\%) \\
\hline \multirow{5}{*}{$\begin{array}{l}\text { Primary } \\
\text { Tocophobia } \\
\text { Social } \\
\text { Factors }\end{array}$} & Culture & $5(5.6)$ \\
\hline & Too weak for labor & $25(27.8)$ \\
\hline & $\begin{array}{l}\text { Family and friends } \\
\text { recommendation }\end{array}$ & $22(24.4)$ \\
\hline & $\begin{array}{l}\text { Feeling of being trauma and } \\
\text { abuse }\end{array}$ & $16(17.8)$ \\
\hline & Anxiety & $22(24.4)$ \\
\hline \multirow{8}{*}{$\begin{array}{l}\text { Fear of Child } \\
\text { Birth }\end{array}$} & \begin{tabular}{|l|l} 
Pelvic floor injury \\
\end{tabular} & $2(1.9)$ \\
\hline & Intense prolonged labor pains & $20(19.2)$ \\
\hline & No pain relief & $6(5.8)$ \\
\hline & Emergency section & $15(14.4)$ \\
\hline & Losing the baby fear & $15(14.4)$ \\
\hline & $\begin{array}{l}\text { Fear of being left alone in } \\
\text { labor }\end{array}$ & $13(12.5)$ \\
\hline & Lack of control & $27(26)$ \\
\hline & Too much waiting time & $6(5.8)$ \\
\hline
\end{tabular}

Secondary tocophobia was faced by multiparous women. The predominant fears in these women were prolonged labor in 15 (37.5\%), fear of labor pains due to lack of pain relief in 11 (27.5\%), birth trauma in $9(22.5 \%)$ and sub optimal fetal outcome in $5(12.5 \%)$. For secondary tocophobia with one previous cesarean section, $37(42 \%)$ ladies preferred cesarean section in next pregnancy because of bearable post operative pain as compared to labor pains. Planned cesarean section was convenient for $20(22.7 \%)$ and 14 (15.9\%) liked complete control over timing of delivery. The factors related to secondary tocophobia are shown in table-III.

For subsequent vaginal deliveries, 19 (20.2\%) asked for pain relief, 9 (9.6\%) preferred an attendant during labor, 31 (33\%) wanted both pain relief and an attendant and $35(37.2 \%)$ did not opt for vaginal delivery. These factors are shown in table-IV.

Table III: Secondary tocophobic factors.

\begin{tabular}{|c|c|c|}
\hline & Factors & n (\%) \\
\hline \multirow{4}{*}{$\begin{array}{l}\text { Secondary } \\
\text { Tocophobia }\end{array}$} & Prolonged labor & $15(37.5)$ \\
\hline & Sub optimal fetal outcome & $5(12.5)$ \\
\hline & Birth trauma & $9(22.5)$ \\
\hline & $\begin{array}{l}\text { Fear of labor pain due to lack } \\
\text { of pain relief }\end{array}$ & $11(27.5)$ \\
\hline \multirow{6}{*}{$\begin{array}{l}\text { Secondary } \\
\text { Tocophobia } \\
\text { for Patients } \\
\text { with one } \\
\text { Previous } \\
\text { C-Section }\end{array}$} & $\begin{array}{l}\text { Emergency c-section directly } \\
\text { correlated to negative } \\
\text { experience of some relatives }\end{array}$ & $13(14.8)$ \\
\hline & $\begin{array}{l}\text { Found timed cesarean section } \\
\text { very convenient }\end{array}$ & $20(22.7)$ \\
\hline & $\begin{array}{l}\text { Complete control over timing } \\
\text { of delivery }\end{array}$ & $14(15.9)$ \\
\hline & Minimal waiting time & $3(3.4)$ \\
\hline & $\begin{array}{l}\text { Bearable post operation pain } \\
\text { compared to labor pain }\end{array}$ & $37(42)$ \\
\hline & Fear of scar rupture & $1(1.1)$ \\
\hline
\end{tabular}

Table-IV: Factors for option of vaginal delivery.

\begin{tabular}{l|c}
\hline Factors & n (\%) \\
\hline Pain Relief & $19(20.2)$ \\
\hline Attendant in Labor Room & $9(9.6)$ \\
\hline Pain Relief+Attendant & $31(33)$ \\
\hline Not opted for Labor & $35(37.2)$ \\
\hline
\end{tabular}

\section{DISCUSSION}

Cesarean section on request is globally on the rise predominantly for social and psychological reasons. The term CSMR was adopted by National Institute of Health state-of-the-science conference in 2006 ${ }^{11}$. They defined CSMR as the primary pre labor cesarean delivery performed on maternal request in the absence of fetal or maternal indications. They reflected that currently the data is not adequate to justify either mode of delivery. 
There is a complex plethora of reasons for which CSMR is performed. The obstetricians face a constant dilemma in decision making in this situation. It is difficult to refuse the request of the patient but at the same time, fetal and maternal risks due to anesthesia and surgery cannot be over looked. The women who undergo cesarean in their first pregnancy are more likely to have cesarean deliveries in subsequent pregnancies ${ }^{12}$. In our study, CSMR was expressed by primigravida $11(9.6 \%)$. Maximum request was from patients with previous cesarean section 83 (72.2\%). Main factors for CSMR in our study were fear of pains and loss of control. Fenwick et alalso found child birth fear and issues of control as the main reasons for CSMR ${ }^{12}$.

The Health Committee Maternity Services and the Changing Childbirth suggest a pivotal role of women indecision making ${ }^{13}$. This view has received criticism. The obstetric decisions should not be affected by maternal choice and fears. Our study aimed to high light the main tocophobic factors that force the women for CSMR.Our objective was to make obstetricians aware of the alarming rise in CSMR and factors that contribute towards it. A North Western Carolina survey concluded that primary reasons for maternal request were prevention of birth injury and existing medical conditions. The primary objective of these women was their infants' health rather their own ${ }^{14}$. In our study, sub optimal fetal outcome was feared by $15(14.4 \%)$ in primary tocophobia and 5 (12.5\%) in secondary tocophobia.

It is difficult to exactly gauge the incidence of tocophobia as women of different levels of tocophobia are usually included in the research. A meta analysis by Connell and colleagues showed the prevalence of $14 \%$. They commented that more research is required to gain a better understanding of fear of child birth ${ }^{15}$. We found in our study that the pain relief 19 (20.2\%) and presence of a partner $31(33 \%)$ were the main requests from those who opted for vaginal delivery. Connell et al, also commented that anxieties, past sexual experience, negative information from friends or relatives, lack of self control were the main factors for primary tocophobia. Secondary tocophobia resulted from a traumatic birth experience, post traumatic stress disorder, birth trauma or sub optimal birth outcome ${ }^{15}$. In addition to these negative thoughts, elite societal and professionally committed ladies preferred to have control over their life events like planning mode and time of delivery. Our findings showed that in primary tocophobia recommendation from family or friends and anxiety were the main determinants. Both seen in
$22(24.7 \%)$ ladies each. For secondary tocophobia, 20 $(22.7 \%)$ found timed cesarean delivery more convenient. Negative experiences from family and friends influenced 13 (14.8\%) secondary tocophobic women.

A study from a tertiary care hospital in Sindh, Pakistan, observed CSMR as the fifth common reason for rise in cesarean section rate ${ }^{16}$. A Swedish registry based study showed that rate of CSMR has increased 3 fold in a ten year period but it did not significantly contribute to the overall cesarean section rate ${ }^{17}$. This study showed that primiparous women requesting CSMR had fear of birth and pain, safety issues, relatives' birth history and history of sexual harassment ${ }^{17}$. This was similar to the reasons expressed by the primary tocophobic women in our study. A Norwegian study documented 10\% CSMR rate which was less than $1 \%$ of all the births at that time ${ }^{18}$. Emma and colleagues studied contributing factors for rising cesarean section rate and found that the rate of cesarean sections on maternal request has risen by $8 \%$ over time $^{19}$. Another qualitative study from Norway found previous birth experience as the major determinant for fear of subsequent births ${ }^{20}$. This finding is consistent with findings of our study.

A cohort of six European countries was studied for preferences of women for mode of delivery. They concluded that medical and psychological concerns are the main determinants behind the maternal request ${ }^{21}$. A Cochrane database review highlighted that there is no substantial evidence for performing cesarean sections for non medical reasons. They have suggested a need for further research in thisregard ${ }^{22}$. Our objective was to high light the womens' choice and fears for mode of delivery and the factors that can modify them. We also feel a need for a further research in CSMR to formulate a plan for tocophobic women and reduce cesarean sections perfomed for non medical reasons.

In their commentary Dweik and Sluijs highlighted that promoting positive birth experience along with healthy mother and child should be the most important goals of the antenatal services to reduce the fear of birth $^{23}$. In a Danish study, maternal request cesarean (MRS) are on the rise. Women who had perineal tears, emergency cesarean and perinatal death had 1.3, 3.8 and 2.0 times more MRS in their next delivery ${ }^{24}$. Prolonged labor and birth trauma were common secondary tocophobic factors in our study. The availability of anesthesia in labor room was the major concern and lack of pain relief was expressed by $27.5 \%$ ladies in our study. A study from Beijing Obstetrics and Gynecology 
Hospital reflected a rise in cesarean section rate in the last twenty years. The changing trend was the rise in cesarean sections for maternal request and previous cesarean delivery.

Our results were comparable with the results of various national and international institutes where rise in CSMR has been highlighted although not a major contributing factor for the total rise in cesarean section rate. Analysis of factors depicted that fear of childbirth, previous birth experience, social recommendations and pain were the main reason behind this rise. Pain relief and availability of an attendant in labor room were the confounding variables which can improve the patient's attitude towards vaginal delivery.

\section{RECOMMENDATION}

Larger studies in both private and public sectors are required to find out the prevalence of CSMR. The demographic, social and psychological reasons need to be evaluated to control the rising trend. Underlying anxiety and stress disorders should be addressed for improved perception of natural child birth.

\section{CONCLUSION}

CSMR has been labeled as an iatrogenic issue with a potential for improvement. A substantial rise has been seen in educated, wealthier, urban women who prefer small family size and convenience of planned delivery. These patients may benefit from more careful surveillance and counseling. Our findings can have significant health implications to control the factors and fears behind CSMR. Obstetricians, lady health workers, counselors and birth attendants need to play their role in alleviating tocophobias. The measures contributing towards acceptance of natural birth should be improved including provision of pain relief and presence of companion in labor rooms.

\section{CONFLICT OF INTEREST}

The study has no conflict of interest to be declared by any author.

\section{REFERENCES}

1. Yajun L, Xin W, Liying Z, Yan R, Weiyuan Z. An analysis of variation sofindications and maternal-fetal prognosis for cesarean section in a tertiary hospital of Beijing: A population based retrospective cohort study. Med Baltimore 2017; 96(7): e5509-12.

2. Abbas F, Amir R, Sadiq M. Prevalence and determinants of Caesarean delivery in Punjab, Pakistan. East Mediterr Health J 2018; 24(11): 1058-69.

3. Nama V, Wilcock F. Cesarean section on maternal request: is justification necessary. Obstetric Gynecol 2011; 13(4): 263-69.

4. Gamble JA, Creedy DK. Women's request for a cesarean section: a critique of the literature. Birth 2000; 27(4): 256-63.

5. Ronsmans C, Graham WJ. Maternal mortality: who, when, where, and why. Lancet 2006; 368(2): 1189-00.

6. ACOG Committee Opinion No. 761: Cesarean delivery on maternal demand. Obstet Gynecol 2019; 133(3): e73-75.

7. Leone T, Padmadas SS, Matthews Z. Community factors affecting rising caesarean section rates in developing countries: an analysis of six countries. Soc Sci Med 2008; 67(8): 1236-46.

8. Sahlin M,Carlander-Klint AK, Hildingsson I, Wiklund I. Firsttimemothers'wishforaplanned caesarean section: deeply rooted emotions. Midwifery 2013; 29(5): 447-52.

9. Wiklund I, Edman G, Andolf E. Cesarean section on maternal request: reasons for the request, self-estimated health, expectations, experience of birth and signs of depression among firsttime mothers. Acta Obstet Gynecol Scand 2007; 86(4): 451-56.

10. Al Mufti R, Carthy A, Fisk NM. Survey of obstetrician's personal preferenceand discretionary practice. Eur J Obstet Gynecol Reprod Biol 1997; 73(1): 1-4.

11. National Institute of Health state-of-the-science conference statement: Cesarean delivery on maternal request. Obstet Gynecol 2006; 107(2): 1386-97.

12. Fenwick J, Staff L, Gamble J, Creedy DK, Bayes S. Why do women request caesarean section in a normal, healthy first pregnancy. Midwifery 2010; 26(4): 394-400.

13. D'Souza R. Caesarean section on maternal request for nonmedical reasons: putting the UK national institute of health and clinical excellence guidelines in perspective. Best Pract Res Clin Obstet Gynaecol 2013; 27(2): 165-77.

14. Romero ST, Coulson CC, Galvin SL. Cesarean birth on maternal request: a western North Carolina perspective. Matern Child Health J 2012; 16(3): 725-34.

15. Connell MA, Warren PL, Kashan A, Kenny LC, O'Neil SM. Worldwide prevalence of tocophobiain pregnant women: systemic review and met analysis. Acta Obstet Gynecol Scan 2017; 96(8): 907-20.

16. Haider G, Zehra N, Munir AA, Haider A. Frequency and indications of cesarean sections in a tertiary care hospital. Pak J Medical Sci 2009; 25(5): 791-96.

17. Karlstrom A, Radestad I, Eriksson C, Rubertsson C, Nystedt A, Hildingsson I. Cesarean section without medical reason, 1997 to 2006: a Swedish register study. Birth 2010; 37(1): 11-20.

18. Kolas T, Hofoss D, Daltveit AK, Nilsen ST. Indications for cesarean deliveries in Norway. Am J Obstet Gynecol 2003; 188(4): 864-70.

19. Barber E, Lundsberg L, Belanger K, Pettker CM, Funai EF, Illuzi JL. Contributing indications to rising cesarean delivery rate. Obstet Gynecol 2011; 118(1): 29-38.

20. Eide KT, Morken HN, Bearoe K. Maternal reasons for requesting planned cesarean section in Norway: a qualitative study. BMC Pregnancy Child Birth 2019; 102(19): 131-40.

21. Ryding EL, Lukasse M, Kristjansdottir H, Steingrimsdottir T, Schei B. Bidens study group. Pregnant women's preference for cesarean section and subsequent mode of birth - a six-country cohort study. J Psychosom Obstet Gynaecol 2016; 37(3): 75-83.

22. Lavender T, Hofmeyr GJ, Neilson JP, Kingdon C, Gyte GM. Caesarean section for non-medical reasons at term. Cochrane Database Syst Rev 2012; 33(2): CD004660-65.

23. Dweik D, Sluijs AM. What is underneath the cesarean request. Acta Obstet Gynecol Scand 2015; 94(11): 1153-55.

24. Forstholm MM, Lidegard O. Cesarean section on maternal request. Ugeskr Laeger 2009; 171(7): 497-02. 